\title{
A new strategy to identify potentially dangerous coronary arterial patterns before percutaneous pulmonary valve implantation
}

\author{
Sebastian Góreczny ${ }^{1}$, Andreas Eicken ${ }^{2}$, Peter Ewert ${ }^{2}$, Gareth John Morgan ${ }^{3}$, Sohrab Fratz ${ }^{2}$ \\ ${ }^{1}$ Department of Cardiology, Polish Mother's Memorial Hospital, Research Institute, Lodz, Poland \\ ${ }^{2}$ Department of Paediatric Cardiology and Congenital Heart Disease, German Heart Center Munich, Technical University Munich, Munich, \\ Germany \\ ${ }^{3}$ Department of Congenital Heart Disease, Evelina London Children's Hospital, London, United Kingdom
}

Postep Kardiol Inter 2014; 10, 4 (38): 294-297

DOI: $10.5114 /$ pwki.2014.46773

\begin{abstract}
A bstract
Despite advances in surgical techniques, right ventricular outflow tract (RVOT) conduits are prone to fail over time. Percutaneous pulmonary valve implantation was introduced to expand the lifetime of these conduits and to decrease the number of open heart operations during a patient's lifetime. The procedure can be performed with excellent results; however, serious complications such as coronary arterial compression and conduit rupture have been reported. We present percutaneous treatment of a patient after Ross-Konno operation with RVOT conduit dysfunction and a potentially problematic course of the left anterior descending artery.
\end{abstract}

Key words: percutaneous pulmonary valve implantation, coronary compression.

\section{Introduction}

Despite advances in surgical techniques, right ventricular outflow tract (RVOT) conduits are prone to fail over time [1]. Patients with surgically reconstructed outflow tracts often require multiple open heart operations during their lifetime. Percutaneous pulmonary valve implantation (PPVI) was introduced to decrease the number of open heart operations and increase the lifespan of conduits by removing obstruction and revalving the surgically placed outflow tube [2]. This procedure can be performed with excellent short- and mid-term results, with low morbidity and mortality [3-5]. One of the most serious potential complications is coronary arterial compression [6, 7]. Preprocedural imaging (computed tomography (CT) or cardiovascular magnetic resonance $(C M R))$, careful patient selection, and a patient-modified approach during the procedure can guide a safe and successful intervention [4]. The large variation in anatomy that exists amongst patients who require RVOT conduits leads to significant diversity in the relationship between the outflow anatomy and the coronary arterial course [8]. Coronary arterial compression, a potentially fatal compli- cation, may occur during PPVI [3, 4, 6, 9]. To minimise the risk of this major adverse event we suggest noninvasive imaging followed by meticulous biplane visualisation of the coronary anatomy. Introduction of a coronary guide wire to the segment of the artery deemed to be a hazard during RVOT conduit expansion may provide further information regarding the risk of coronary compression.

We present the findings of a patient after Ross-Konno operation with dysfunction of the RVOT conduit. In CMR a potentially dangerous course of the left anterior descending (LAD) artery was noticed. The balloon test was done with concomitant placement of a coronary guidewire to exclude a compression of the LAD during PPVI.

\section{Case report}

A 15-year-old boy $(177 \mathrm{~cm}, 97 \mathrm{~kg})$ presented with mixed stenotic and regurgitant dysfunction of an RVOT conduit. Following an initial neonatal aortic balloon valvuloplasty a Ross-Konno operation was performed in infancy. Five years later the initial RVOT conduit (pulmonary homograft) was replaced with a $20 \mathrm{~mm}$ bovine xenograft conduit (Contegra, Medtronic Inc, Minneapolis, MN).

\section{Corresponding author:}

Sebastian Góreczny MD, PhD, Department of Cardiology, Polish Mother's Memorial Hospital, Research Institute, $281 / 289$ Rzgowska St, 93338 Lodz, Poland, phone: +48 4227121 84, e-mail: sebastiangoreczny@yahoo.pl

Received: 13.08.2014, accepted: 29.09.2014. 
The patient presented in good clinical condition with typical ECG and echocardiographic findings of RVOT conduit dysfunction. The maximal RVOT velocity (Vmax, continuous wave Doppler) was $4.2 \mathrm{~m} / \mathrm{s}$, with proximal stenosis of the conduit and significant pulmonary regurgitation. Cardiovascular magnetic resonance demonstrated good global biventricular function without regional wall disturbances and moderate right ventricular hypertrophy. The RV was mildly dilated with an indexed end diastolic volume of $88 \mathrm{ml} / \mathrm{m}^{2}$. The coronary artery origins were in the usual positions. The right coronary artery was far from the conduit, whereas the LAD branch coursed parallel to and postero-inferior to the conduit at a distance of approximately $4 \mathrm{~mm}$ (Figures $1 \mathrm{~A}-\mathrm{D}$ ). This arrangement was deemed to be potentially high risk for coronary compression therefore further interrogation was performed.

At cardiac catheterisation under general anaesthesia, angiography delineated a tight proximal stenosis of the conduit with a minimal diameter of $11 \mathrm{~mm}$. Moderate calcifications around the proximal segment of the conduit were noted (Figures 2 A, B). Haemodynamic measurements were performed (right ventricle 52/5/13 mm Hg, pulmonary artery - 23/12/15 mm Hg, aorta - 85/62/79 $\mathrm{mm} \mathrm{Hg}$ ). For balloon testing an $18 \mathrm{~mm}$ low-pressure balloon (BiB, NuMed Inc, Hopkinton, NY) was inflated in the RVOT with concomitant selective left coronary angiography in posterior-anterior and left lateral projections. As depicted by CMR, the LAD branch coursed at a close distance to the proximal calcified part of the conduit, creating a potential risk of compression in case of expansion. No evidence of coronary compression was seen on angiography or on ECG analysis at this point. To better understand the relationship along the proximal course of the LAD and the conduit, a soft tipped 0.014 inch standard coronary guide wire was advanced into the LAD to a point where it looked to be closest to the con-
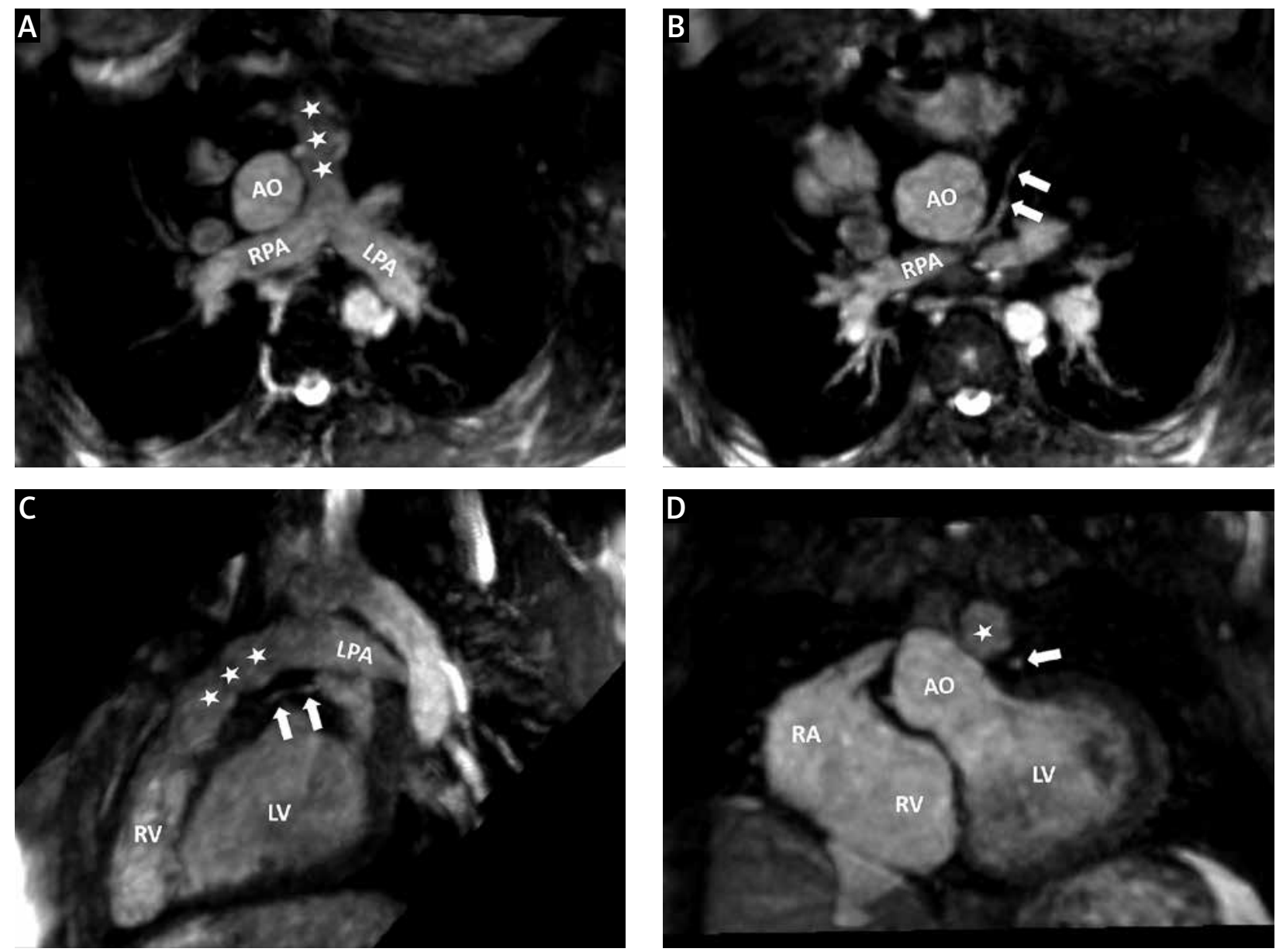

Figure 1. ECG-triggered and respiratory navigator-gated 3D balanced Steady-State Free Precision CMR images. A - Para-axial plane on the level of main pulmonary artery bifurcation, $\mathbf{B}$ - plane slightly caudal on the level of left coronary artery origin and the proximal segment of the LAD (white arrows) branch, $\mathrm{C}$ - para-sagittal and D - coronal planes. The LAD branch courses directly beneath and parallel to the narrow conduit (white stars) creating potential conflict in case of expansion of the conduit

$C M R$ - cardiovascular magnetic resonance, $L A D$ - left anterior descending, AO - ascending aorta, LPA - left pulmonary artery, RPA - right pulmonary artery, $R V$ - right ventricle, $R A$ - right atrium, $L V$ - left ventricle 

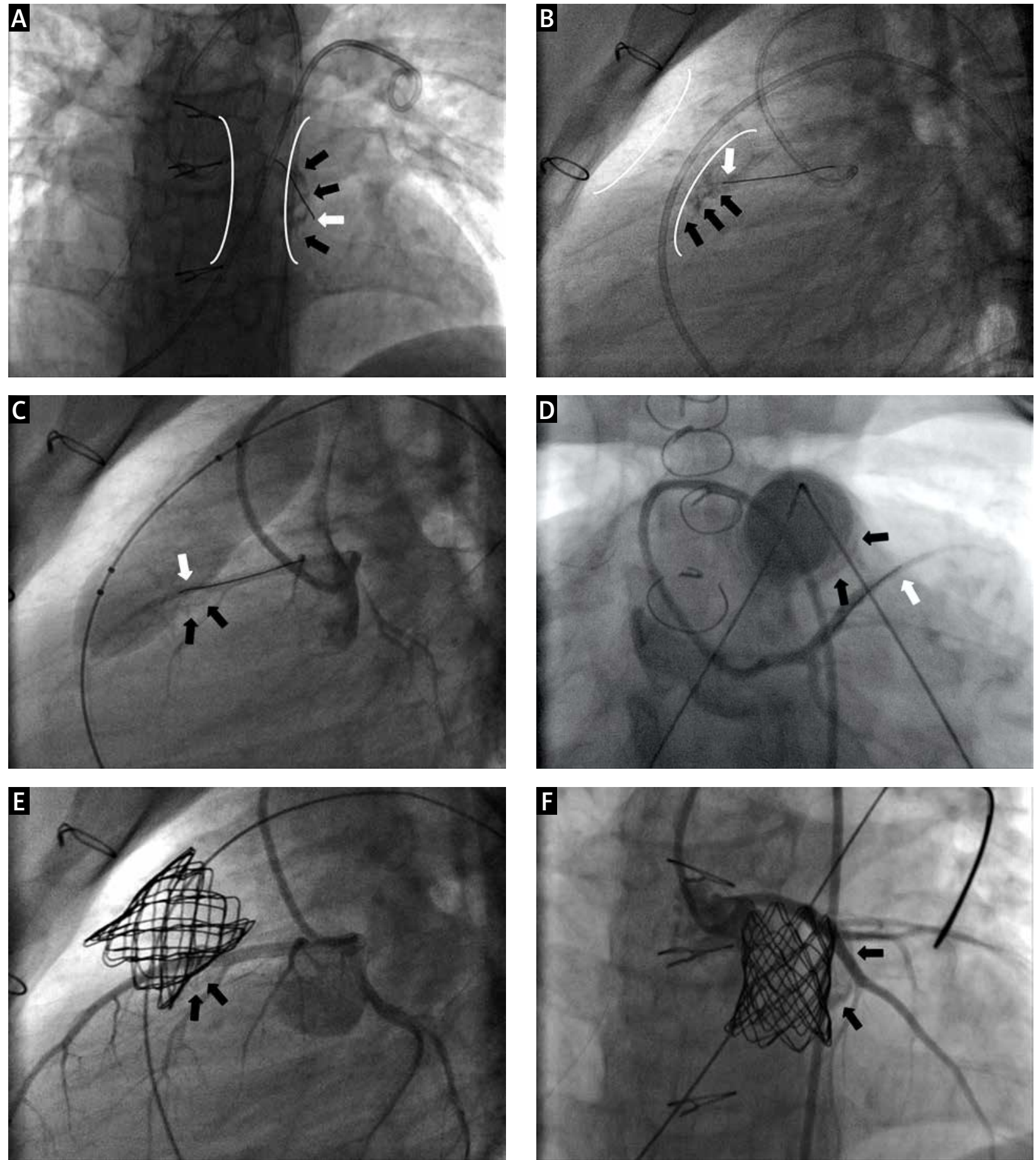

Figure 2. Step-by-step angiographic images of PPVI. Standard posterior-anterior (PA) (A) and left lateral (LL) (B) projections showing relationship between the narrow conduit (white curved lines), calcifications (black arrows) in the proximal segment of the conduit, and the proximal segment of the left anterior descending (LAD) branch exposed with the aid of the tip of the coronary guidewire (white arrow). Note the hazardous proximity between the proximal part of the LAD branch and the conduit. The LL (C) and the 'barrel-view' projection (D) during balloon testing with $20 \mathrm{~mm}$ balloon catheter. On LL view, fully inflated balloon seems to project on the tip of the guidewire; however, in the barrel-view projection the tip of the guidewire turns away from the balloon and proximal calcifications. Final selective left coronary angiography in PA (E) and LL (F) projections showing unobstructed flow to all branches 
duit (Figures 2 A, B). Selective biplane left coronary artery angiography was repeated in a left lateral and a frontal $40^{\circ}$ caudal angulation (the so-called "barrel" projection), during inflation of a $20 \mathrm{~mm}$ BiB catheter (Figures $2 \mathrm{C}, \mathrm{D}$ ). In the lateral plane the tip of the guidewire projected over the proximal part of the balloon; however, in the barrel view it was clearly leftward from the conduit. This was deemed to be reassuring and the decision was made to implant the percutaneous pulmonary valve (Melody, Medtronic Inc, Minneapolis, MN). After preparing a landing zone with a $34 \mathrm{~mm}$ long covered Cheatham Platinum (cCP) stent (NuMed Inc) mounted on a $20 \mathrm{~mm}$ BiB catheter, the percutaneous pulmonary valve was implanted on a $20 \mathrm{~mm}$ Ensemble delivery system (Melody, Medtronic Inc, Minneapolis, MN). The whole assembly was post-dilated with a $20 \mathrm{~mm}$ high-pressure balloon catheter (Mullins, NuMed Inc) before control angiography of the left coronary artery showed unobstructed flow in all branches (Figures 2 E, F). Haemodynamic measurements confirmed significant reduction of the RVOT gradient (residual gradient $7 \mathrm{~mm} \mathrm{Hg}$ ) and right ventricle $(39 / 9 / 12 \mathrm{~mm} \mathrm{Hg})$ to systemic pressure $(116 / 71 / 89 \mathrm{~mm} \mathrm{Hg})$ ratio. The patient was discharged home on the second day post intervention.

\section{Discussion}

Despite advances in surgical techniques, RVOT conduits fail over time [1]. Percutaneous pulmonary valve implantation has provided a major step forward in reducing the number of "re-do" surgical conduit replacements during a patient's life [2]. This procedure can be performed with a low morbidity and mortality and excellent medium-term results [3-5]. Along with conduit rupture, coronary compression remains a major potential complication [6-8]. Careful patient selection should include preprocedural imaging (CT and or CMR imaging), with angiographic coronary assessment in selected cases. Balloon interrogation of the conduit with concomitant selective biplane coronary arteriography is invaluable in some cases, but the aggression of the balloon interrogation needs to be balanced by the risk of conduit rupture during this diagnostic step. The PPVI operators should have criteria and protocols for pre-procedure assessment and be prepared to tailor their approach depending on the surgical anatomy. There is no clear method of predicting how a conduit will behave on aggressive expansion and how adjacent structures, including the coronary arteries, will be affected. Opinions vary from expert to expert regarding the "safe distances" between coronary and conduit, the behaviour of soft tissues versus calcified structures, the position of the conduit with respect to the sternum, and how the proposed degree of conduit expansion might predict the amount of excursion of the expanded conduit. All have been touted as determinants of risk of coronary compression [9]. Our approach of combin- ing angiography with the positioning of a coronary wire allows increased information linking the position of the conduit narrowing to the course of the coronary artery. It does not in itself prevent compression but may help predict the likelihood of compression in concerning anatomy. Although major complications during PPVI are rare, we must remember that the risk of surgical conduit replacement in most cases is very low. Our tolerance of risk in these alternative interventional procedures must take this into account in each individual case.

\section{References}

1. Dearani JA, Danielson GK, Puga FJ, et al. Late follow-up of 1095 patients undergoing operation for complex congenital heart disease utilizing pulmonary ventricle to pulmonary artery conduits. Ann Thorac Surg 2003; 75: 399-410.

2. Bonhoeffer P, Boudjemline Y, Qureshi SA, et al. Percutaneous insertion of the pulmonary valve. J Am Coll Cardiol 2002; 39: 1664-9.

3. Lurz P, Coats L, Khambadkone S, et al. Percutaneous pulmonary valve implantation: impact of evolving technology and learning curve on clinical outcome. Circulation 2008; 117: 1964-72.

4. Eicken A, Ewert P, Hager A, et al. Percutaneous pulmonary valve implantation: two-centre experience with more than 100 patients. Eur Heart J 2011; 32: 1260-5.

5. Ruzyłło W, Demkow M, Włodarska EK, et al. POL-PAVTI - Polish report on transcatheter pulmonary artery valve implantation of Melody-Medtronic prosthesis in the first 14 patients in Poland. Kardiol Pol 2009; 67: 1155-61.

6. Sridharan S, Coats L, Khambadkone S, et al. Transcatheter right ventricular outflow tract intervention: the risk to the coronary circulation. Circulation 2006; 113: e934-5.

7. Demkow M, Rużyłło W, Biernacka EK, et al. Is the "porcellain homograft" a contraindication for transcatheter pulmonary valve implantation? A case report. Postep Kardiol Inter 2011; 7: 323-6.

8. Brown JW, Ruzmetov M, Vijay P, et al. The Ross-Konno procedure in children: outcomes, autograft and allograft function, and reoperations. Ann Thorac Surg 2006; 82: 1301-6.

9. Morray $\mathrm{BH}, \mathrm{McElhinney} \mathrm{DB}$, Cheatham JP, et al. Risk of coronary artery compression among patients referred for transcatheter pulmonary valve implantation: a multicenter experience. Circ Cardiovasc Interv 2013; 6: 535-42. 